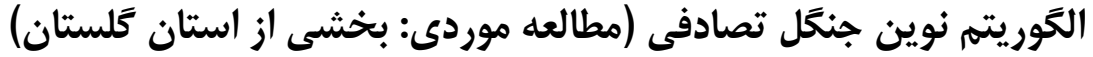

\author{
مجيد محمدى ' و حميدرضا بورقاسمى 'ان
}

ا- استاديار گروه مرتع و آبخيزدارى، دانشكده مهندسى منابع طبيعى، دانشخاه سمنان، (نويسنده مسوول: (majid.mohammady@ @emnan.ac.ir)

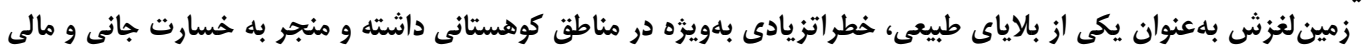

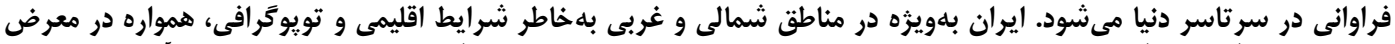

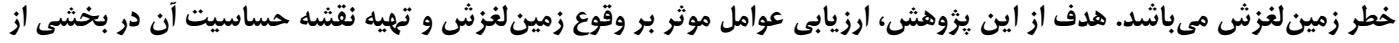

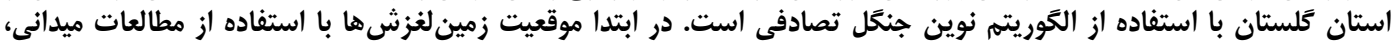

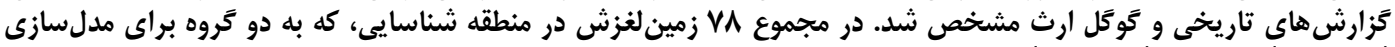

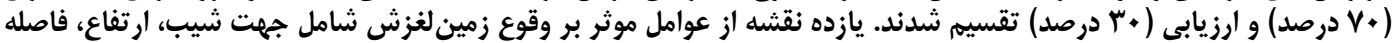

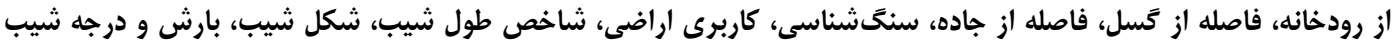

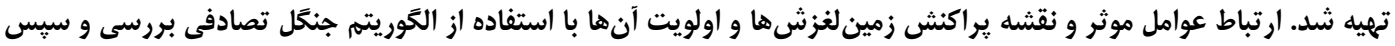

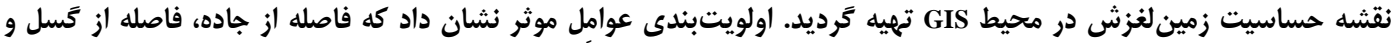

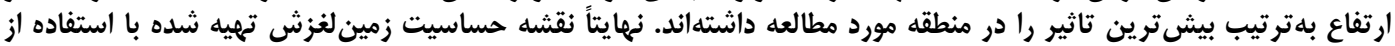

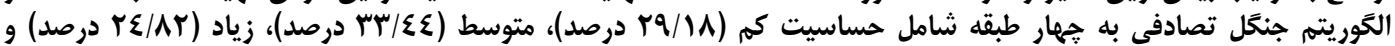

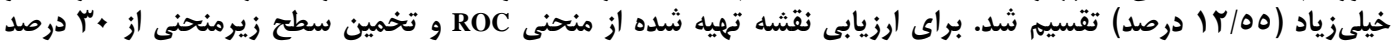

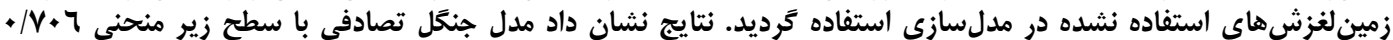
دقت قابل قبولى براى تهيه نقشه حساسيت زمين لغزش استف ارائه كرده است.

وازمهاى كليدى: زمين لغزش، نقشه حساسيت زمين لغزش، سامانه اطلاعات جغرافيايى، الكَوريتم جنكَل تصادفى

ضرورىاست. هدف از تهيه نقشههاى حساسيت زمين لغزش

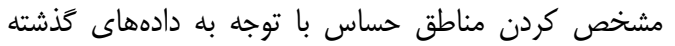

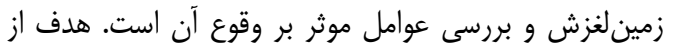

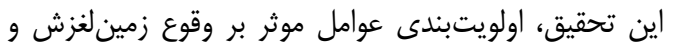

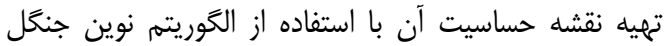
تصادفى در بخشى از مناطق كوهستانى استان كلمتان آنان است.

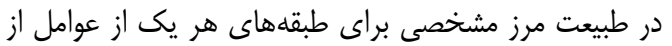
قبيل نوع خاك، انواع كاربرى اراضى و و واحدهاى سنى سنى

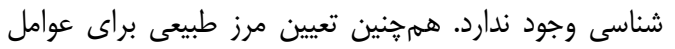

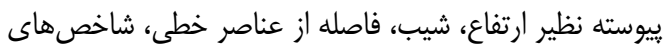

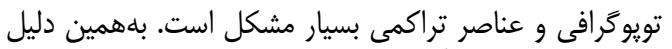

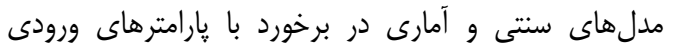

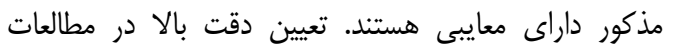

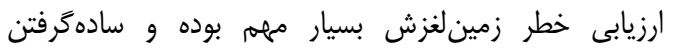

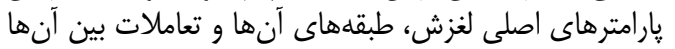

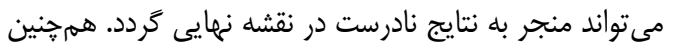

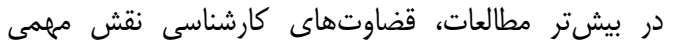

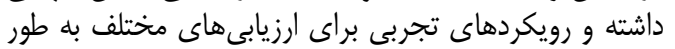

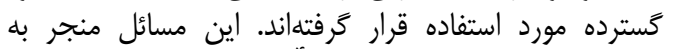

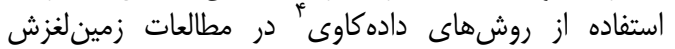

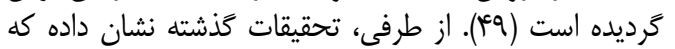

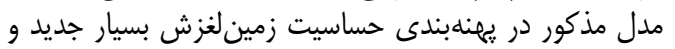

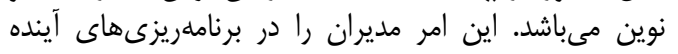

مقدمه - م زمين لغزش موجب خسارت به زندكى و دارايىهاى انسان

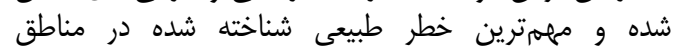

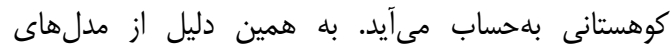

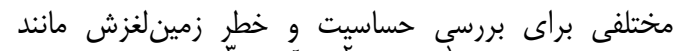

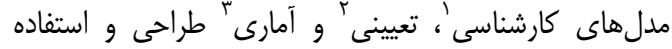

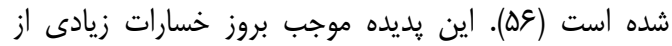

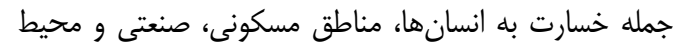

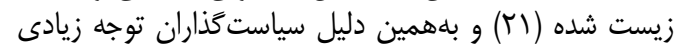

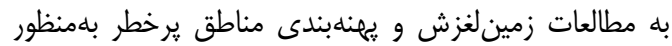

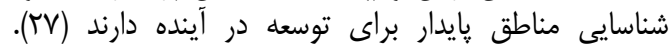

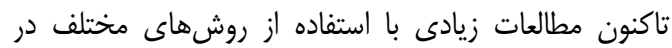

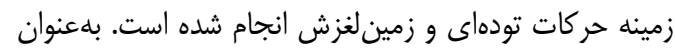

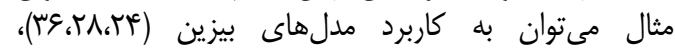

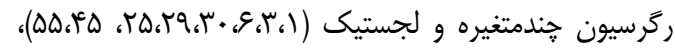

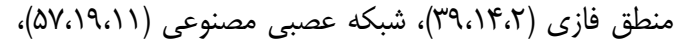

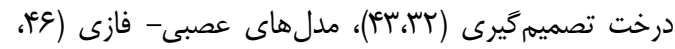

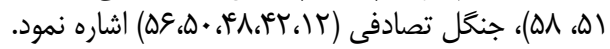

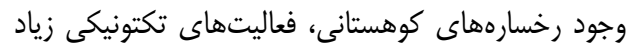

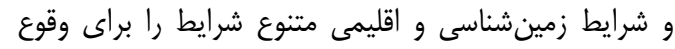

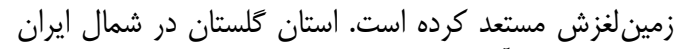

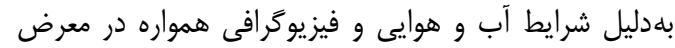
خطر زمينلغزش بوده و و توجه به ائه اين عارضه امرى دمرض

3- Statistical 4- Data Mining


است. نقشه زمينشناسى منطقه مورد مطالعه از سازمان

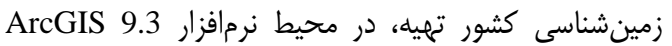

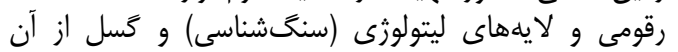

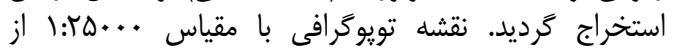

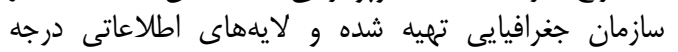

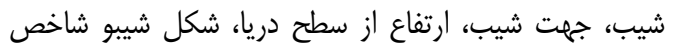

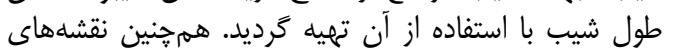

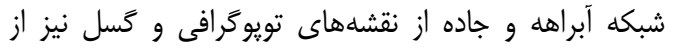

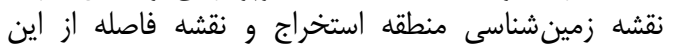

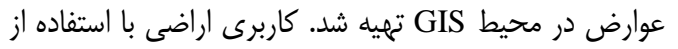

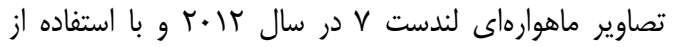

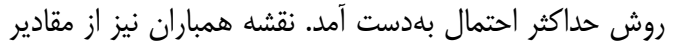

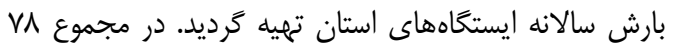

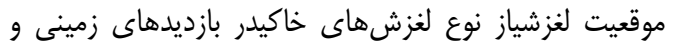

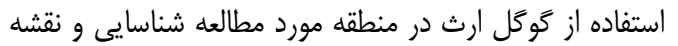

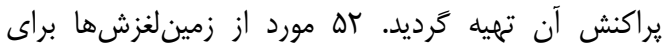
مدلسازى و عا مورد براى ارزيابى مدل در نظر كرفته شد.
كمك نموده تا از تغييرات و دخالتهاى انسانى در اين مناطق مداطق

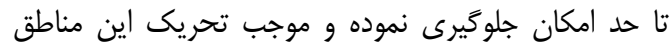
نشود. تا حل

\section{مواد و روشها منطقه مورد مطالعه}

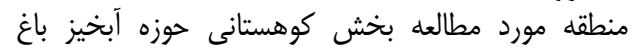

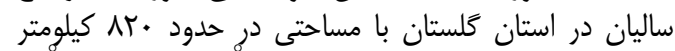

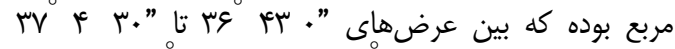

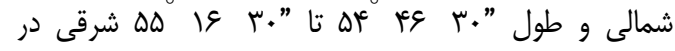

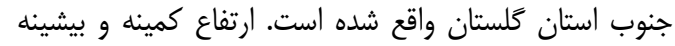

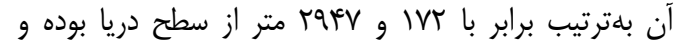

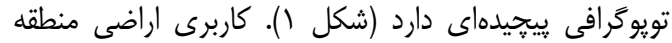

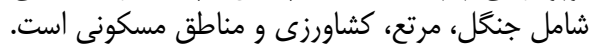

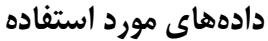

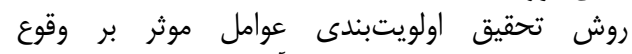
زمينلغزش و تهييه نقشه حساسيت آن با استفاده از الكَوريته جنگل تصادفى بلصورت نمودار جريانى در شكل rا ارائه شده الثران

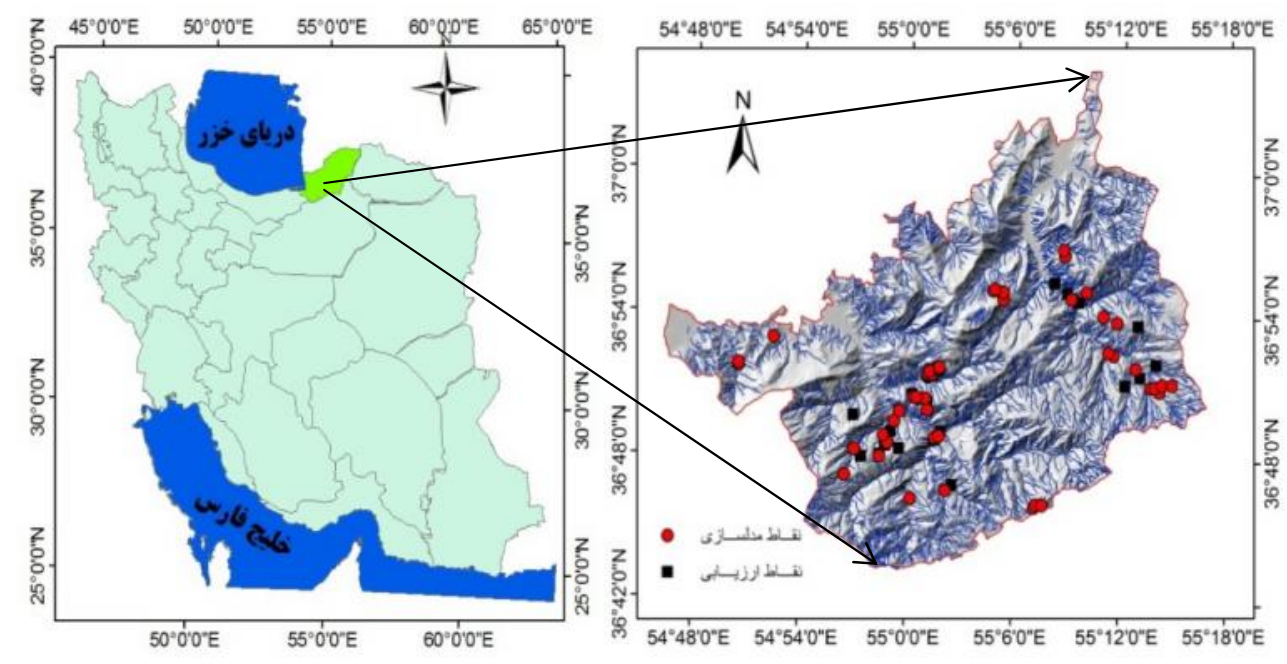

شكل 1- موقعيت منطقه مورد مطالعه

Figure 1. Location of the case study 


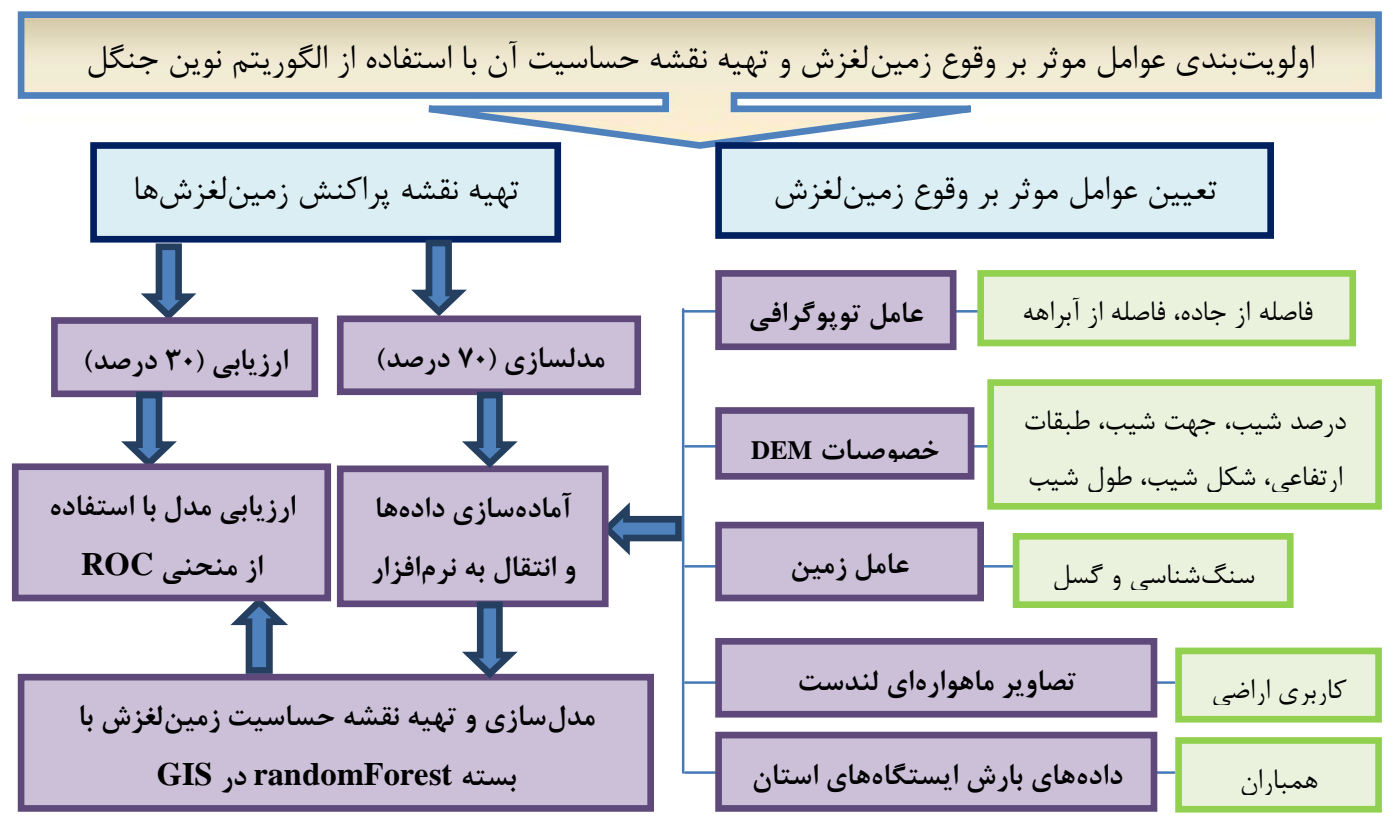

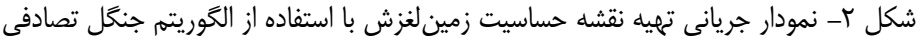

Figure 2. Flowchart of landslide susceptibility mapping using random forest algorithm

در اين مدل از دو عامل ميانكَين كاهشى دقت و ميانكين

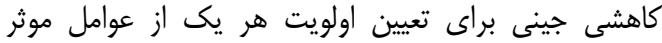

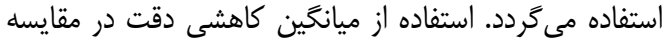

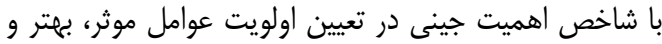

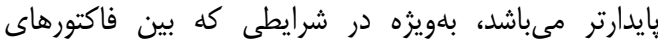

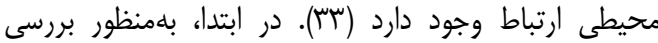

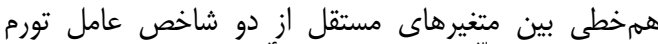

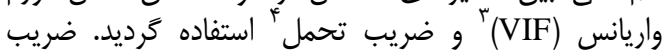

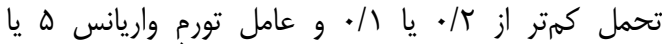

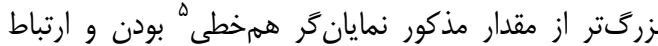

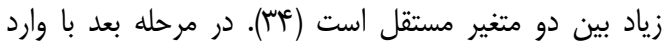

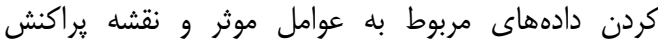

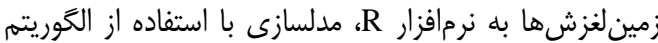

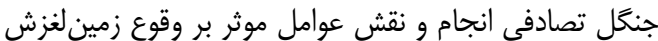

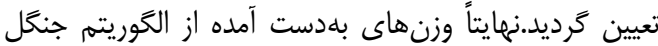

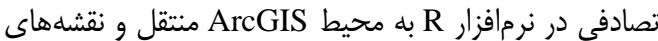

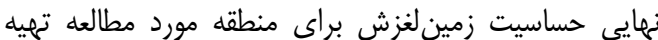

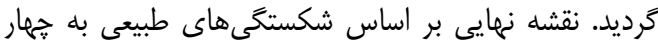

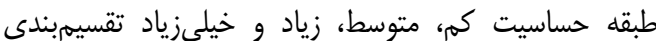

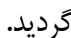

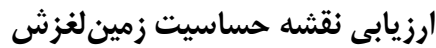

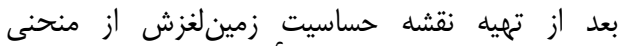

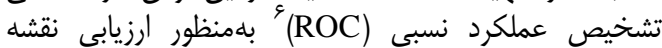

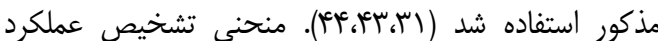

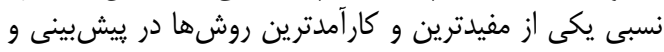

1- Random Forest (RF) 2- Grow

4- Tolerance 5- Multi-Collinearity

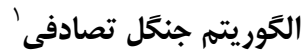

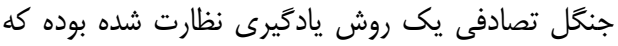

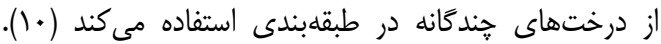

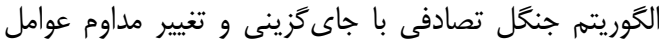
موثر و مرتبط با هدف، منجر به به ايجاد تعداد زياديادى درئ درخت

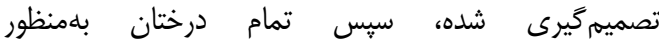

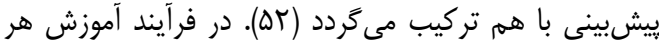

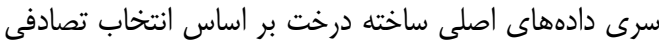

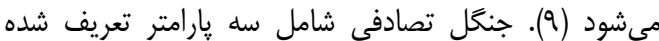

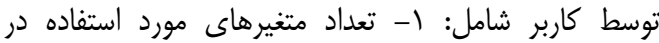

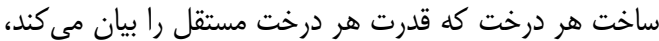

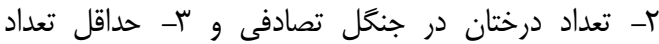

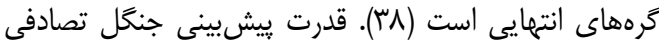

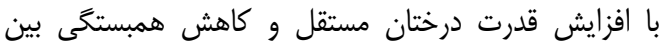

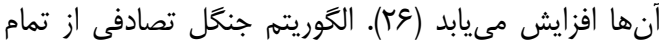

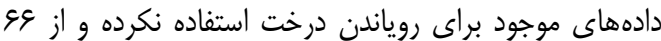

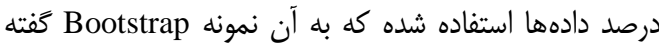

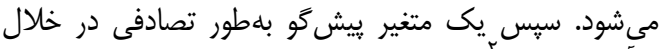

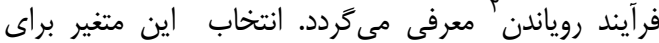

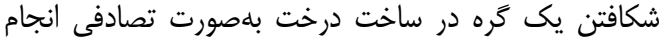

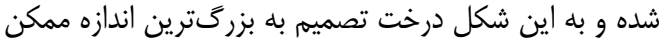

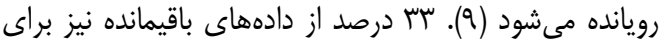

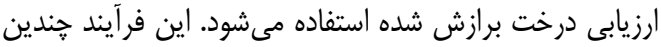

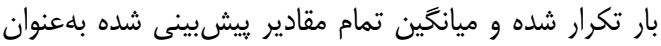

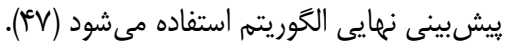
3- Variance Inflation Factor (VIF) 6- Receiver Operating Characteristic (ROC) 
ميانكين كاهشى دقت معيار مناسبترى براى اولويتبندى

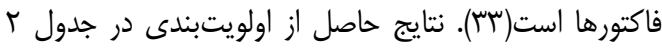

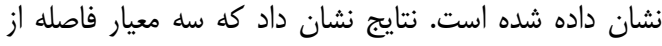

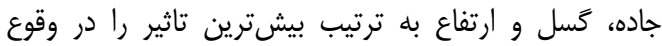

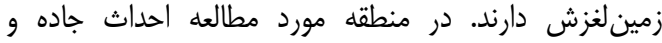

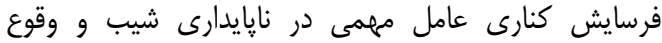

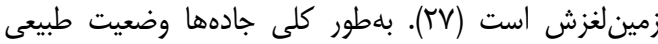

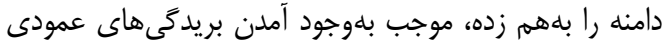

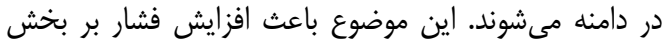

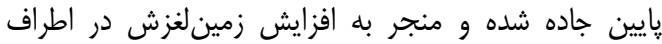

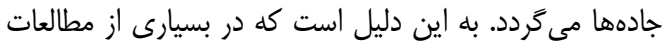

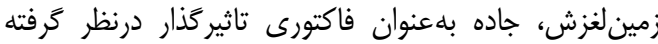

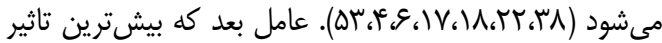

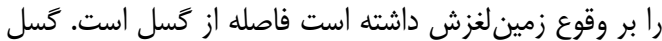

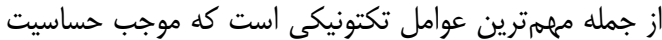

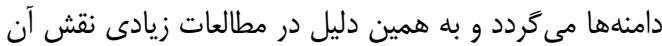

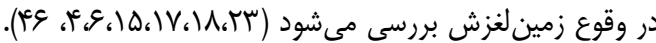

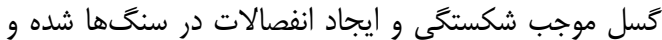

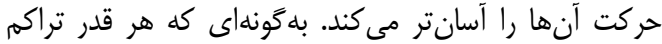

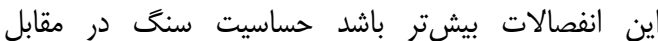

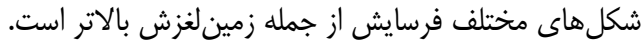

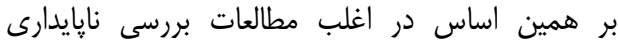

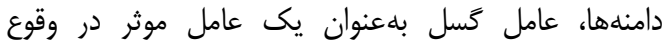
زمين لغزش مورد بررسى قرار مى كيردان
تعيين دقت مدلسازى است و در حقيقت يك نمايش

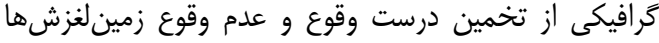

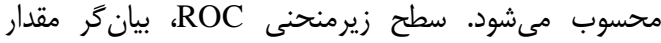

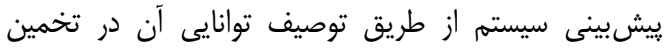

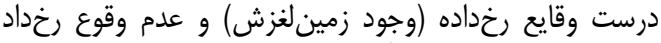

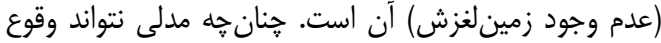

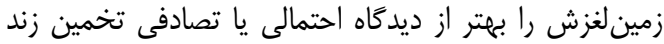

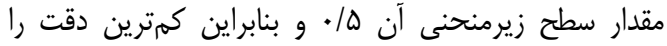

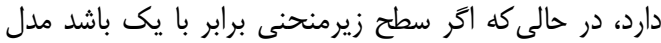

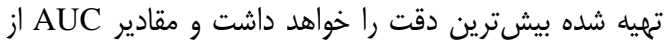

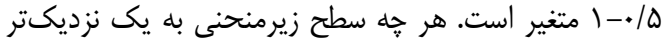

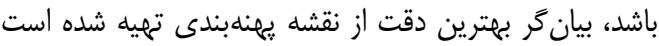

\section{نتايج و بحث}

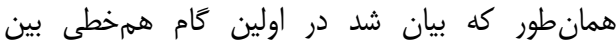

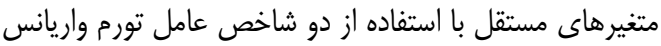

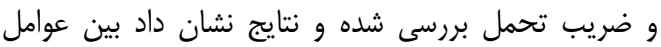

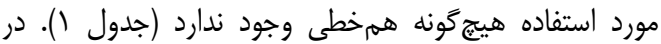

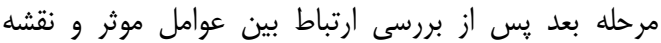

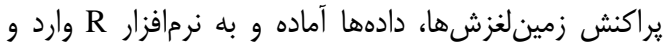

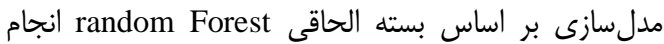

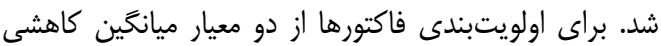

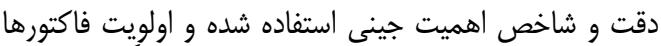

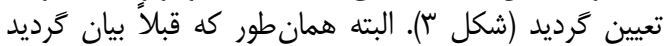

جدول ا- بررسى همخطى بين عوامل موثر بر وقوع زمين لغزش منطقه مورد مطالعه Table 1. Investigating multi-collinearity of landslide conditioning factors in the case study

\begin{tabular}{|c|c|c|}
\hline ضريب تحمل (Tolerance) & عامل تورم واريانس (VIF) & عوامل موثر بر وقوع زمينلغزش \\
\hline - /AFF & I/\AD & جهت شيب \\
\hline$\cdot / 0 \cdot r$ & 1/919 & ارتفاع (متر) \\
\hline.$/ 9$. & $1 / 111$ & فاصله از رودخانه (متر) \\
\hline - /ATE & 1/r/T & زمين شناسى \\
\hline$\cdot / V \Delta$ & 1/ l & 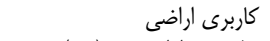 \\
\hline • & $F / I V \Lambda$ & شاخص طول شيب (متر) \\
\hline - /AFF & $1 / 199$ & شكل شيب \\
\hline.$/ 911$ & $1 / 91 \mathrm{~V}$ & بارش سالانه (ميلىمتر) \\
\hline ./19V & $1 / 110$ & فاصله از جاده (متر) \\
\hline - KFq & $\% / .11$ & درجه شيب \\
\hline$\cdot 19 \cdot 0$ & 1/9QT & فاصله از گسل (متر) \\
\hline
\end{tabular}

جدول r- ميزان اهميت عوامل موثر بر وثوع زمين لغزش بر اساس دو معيار ميانخين كاهشى دقت و ميانخين كاهشى جينى Table 2. Importance of conditioning factors based on mean decrease accuracy and mean decrease Gini

\begin{tabular}{|c|c|c|c|c|}
\hline ميانخين كاهشى جينى & ميانخين كاهشى دقت & 1 & $\cdot$ & عامل \\
\hline T/AT & $9 / 9 V$ & $1 \cdot / \Delta V$ & $r / . \varphi$ & جهت شيب \\
\hline$\% / .9$ & $I T / A F$ & $\mid r / A F$ & $8 / 49$ & ارتفاع \\
\hline 1/9r &.$-|\Delta|$ & $1 / 9 V$ & $-r / \Lambda$. & فاصله از رودخانه \\
\hline$r / 91$ & $1 \cdot / \pi 1$ & $11 / \pi r$ & $r / \Delta r$ & ن ش شناسى \\
\hline . & $r / 19$ & $\Gamma / \Lambda \mu$ & 1/r & كاربرى اراضى \\
\hline$r / M$ & $\Delta / 1 \Delta$ & $\Delta / \Gamma$. & 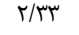 & شاخص طول شيب \\
\hline r/৭9 & $N / T \Delta$ & S/QT & $s / \Delta \omega$ & شكل شيب \\
\hline$r / 4$. & $1 . / 98$ & س & ת Tr/T & بارش سالانه \\
\hline זוא & $T V / r q$ & $r \mu / 19$ & $r .18 \Lambda$ & فاصله از جاده \\
\hline$T / \pi \Delta$ & $r / 9 T^{6}$ & $1 / T \Delta$ & $r / v$. & درجه شيب \\
\hline$\Delta / \Delta T$ & $r \Delta / r \omega$ & $r \mu / q$. & $|V / \Delta|$ & فاصله از گسل \\
\hline
\end{tabular}



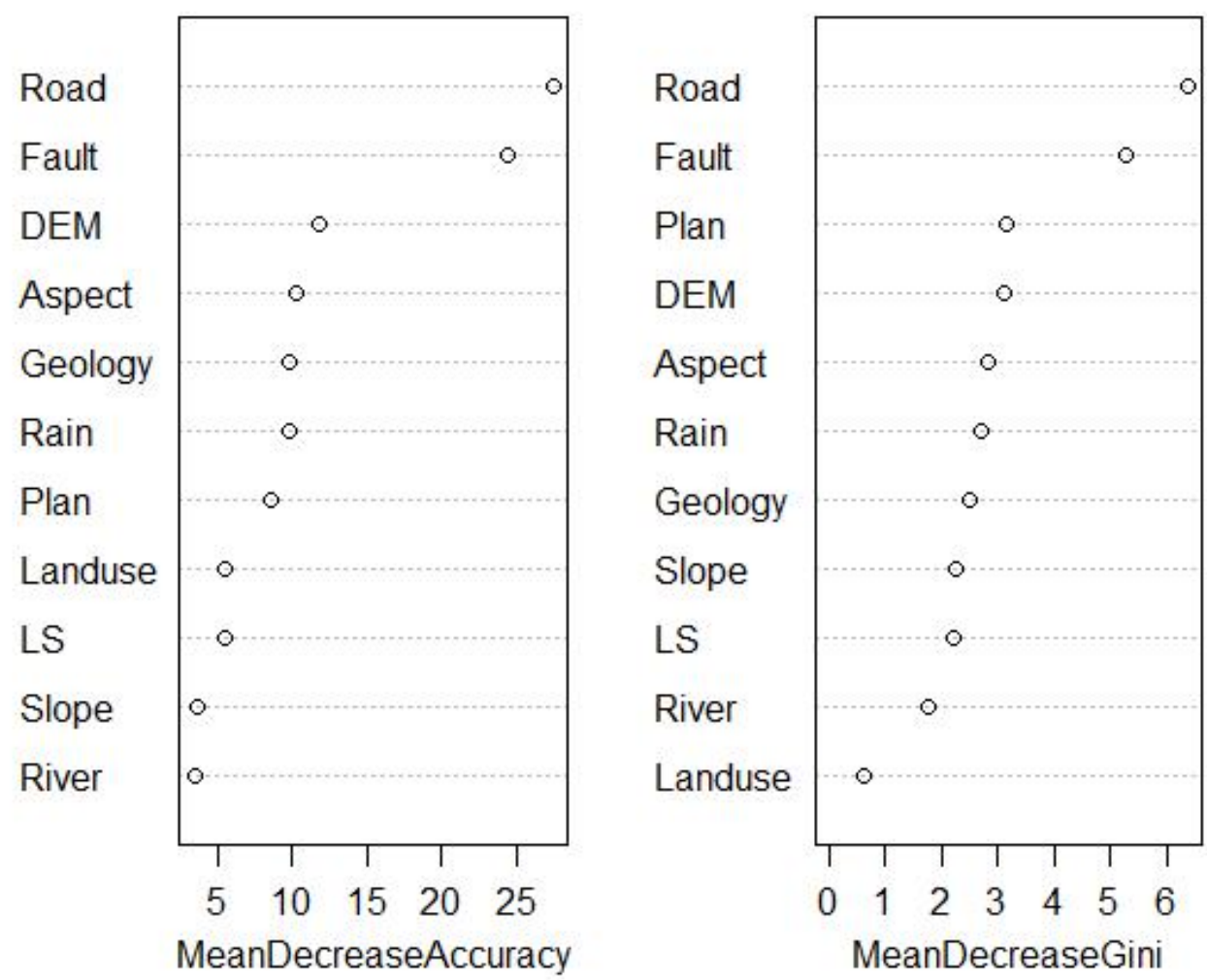

شكل س- اولويتبندى عوامل موثر بر وقوع زمينلغزش با استفاده از الخوريته جنگل تصادفى

Figure 3. Prioritization of landslide conditioning factors using random forest algorithm

بيان

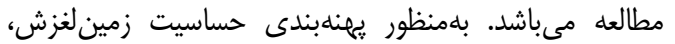

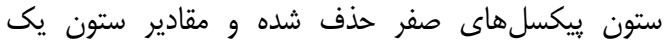

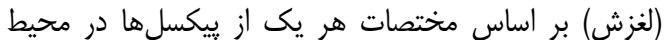
GIS

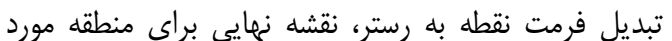

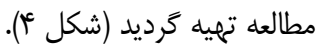

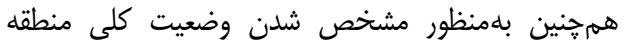
مورد مطالعه، مساحت طبقات حساسيت زمين لغزش محات محاسبه

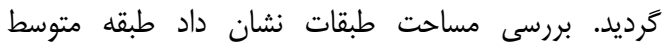

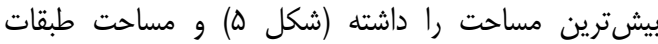
حساسيت زياد و خيلىزياد كرديده است.
عامل موثر ديخر در وقوع زمينلغزش ارتفاع از سطح دريا

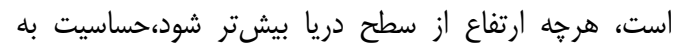

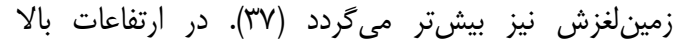

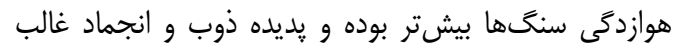

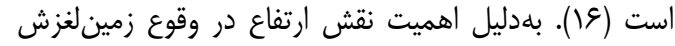

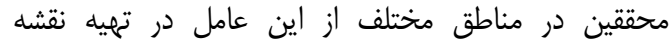

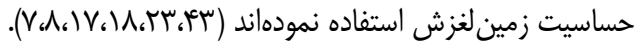

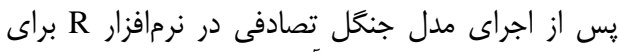

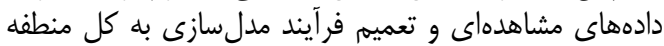

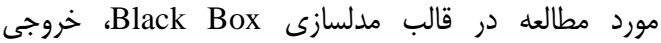

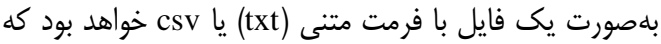

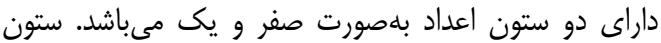

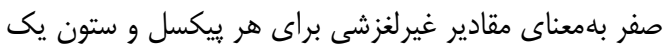




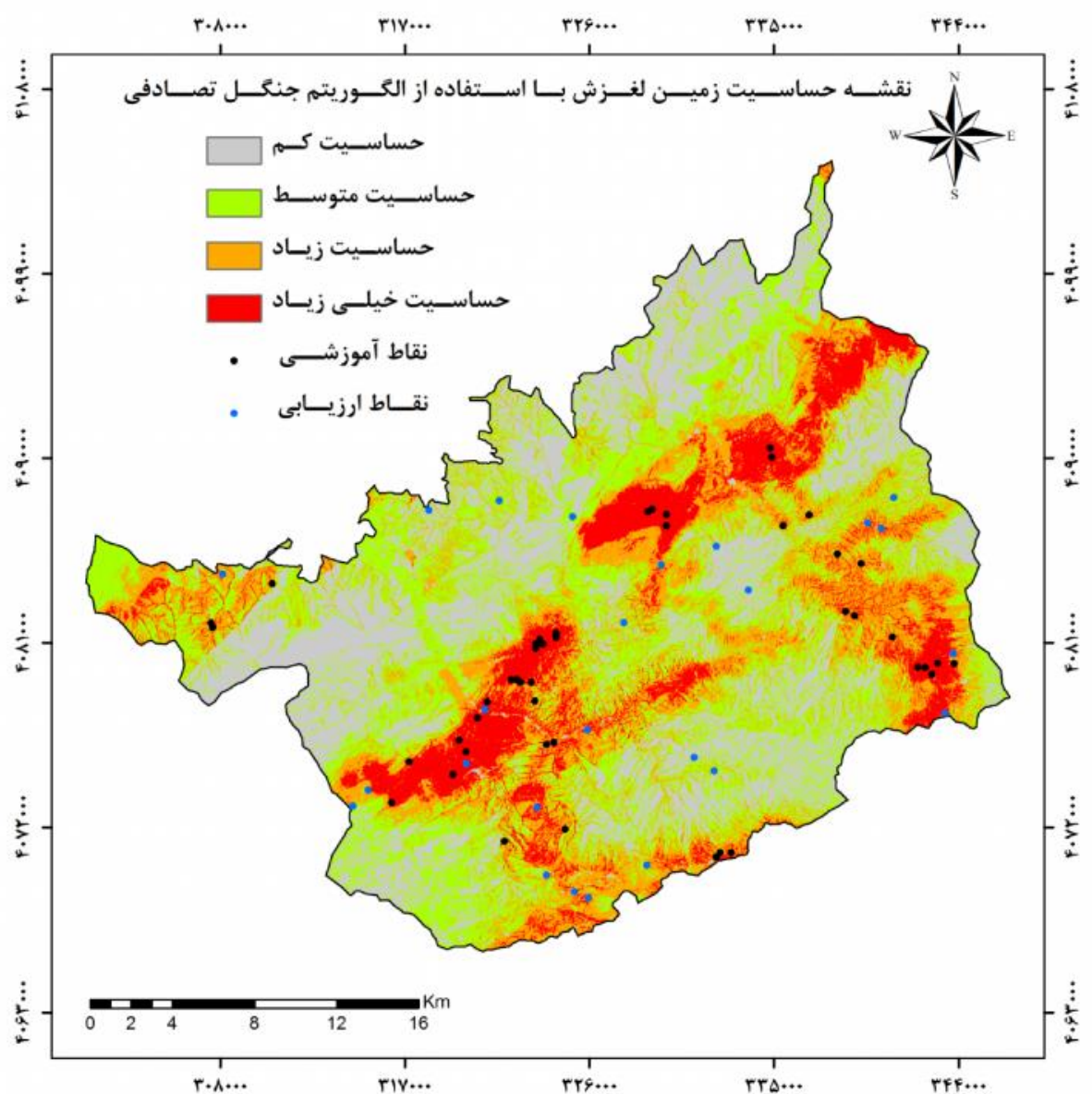

شكل عا- نقشه يهنابندى حساسيت زمين لغزش با استفاده از الكوريتم جنگل تصادفى

Figure 4. Landslide susceptibility map using random forest algorithm

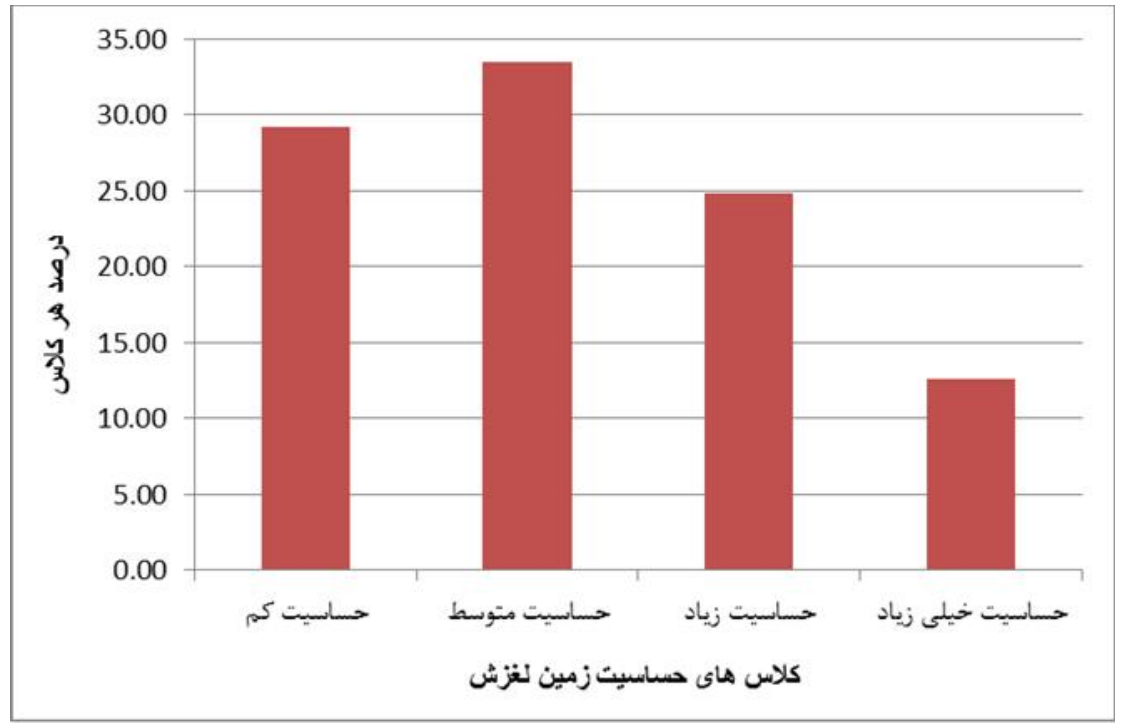

شكل ه- درصد طبقات حساسيت نقشه زمين لغزش در منطقه مورد مطالعه

Figure 5. Percent of landslide susceptibility classes in the case study 
تبديل شده است. استان كَلستان و منطقه مورد مطالعه نيز

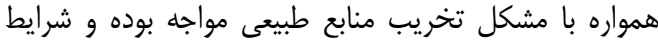

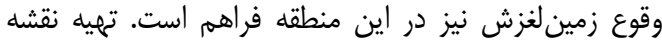

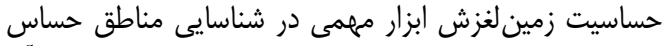

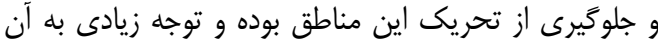

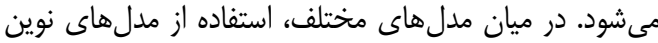
نظير جنكل تصادفى و تلفيق آن بان آنا

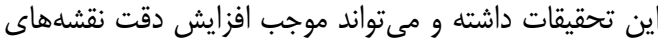

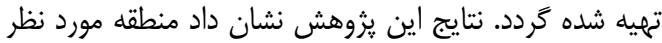

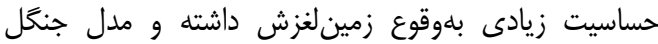

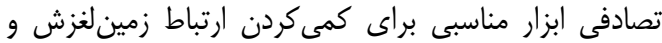

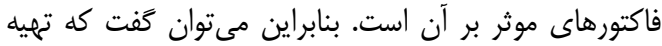

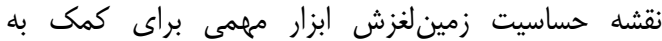

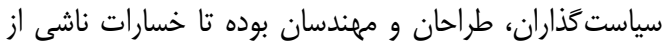
وقوع زمين لغزش را كاهش دهندان
همان طور كه بيان شد براى ارزيابى مدل از منحنى ROC منحئ

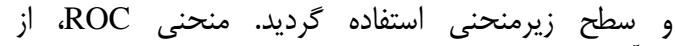
كارآمدترين روشها در ارائه خصوصيت تعيينى، شناسيى مايى

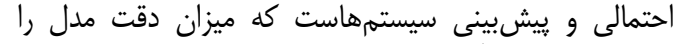

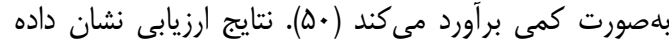

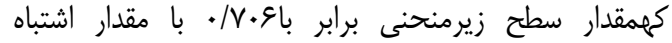

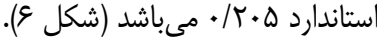

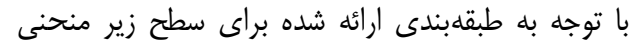

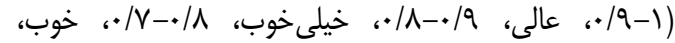

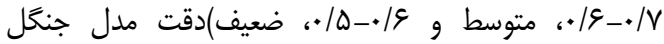

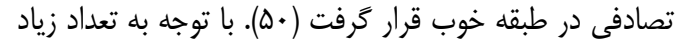

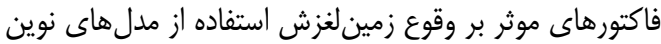

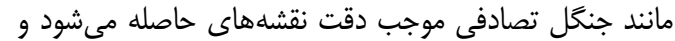

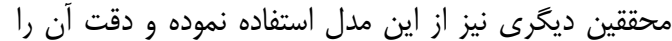

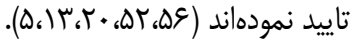

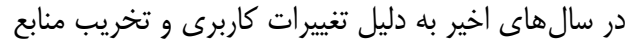
طبيعى، زمين لغزش به خطرى بزرى در در بسيارى از مناطق دنيا

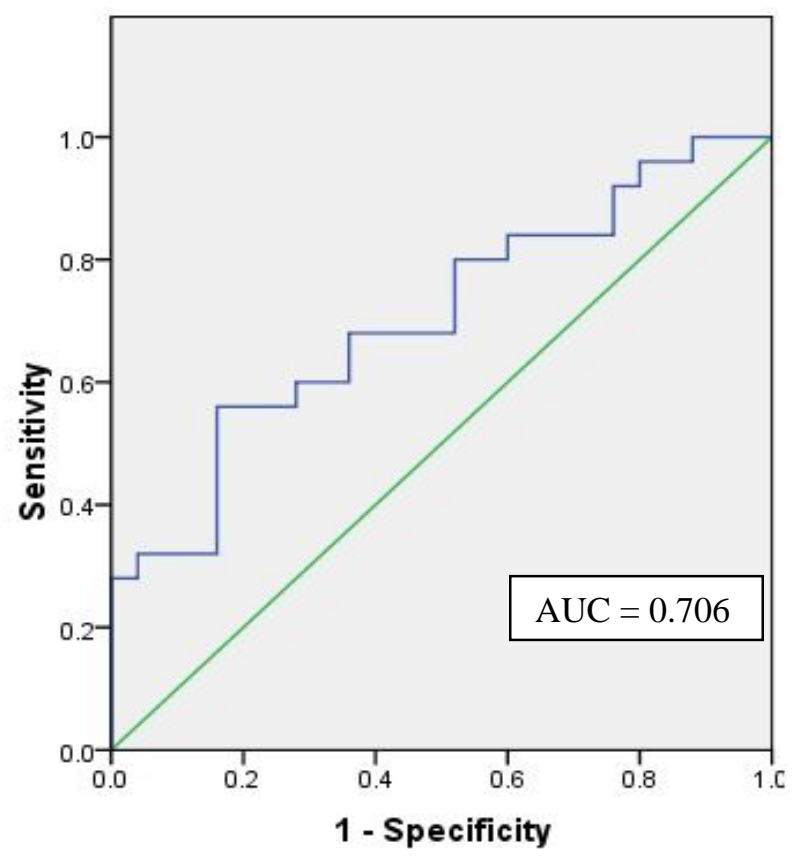

شكل \&- منحنى تشخيص عملكرد نسبى و سطح زير منحنى مربوط به نقشه يهنهبندى حساسيت زمين لغزش

Figure 6. Receiver operating characteristic and area underthe curve of landslide susceptibility map 
1. Akgun, A. C. Kincaland and B. Pradhan 2011. Application of remote sensing data and GIS for landslide risk assessment as an environmental threat to Izmir city (West Turkey). Environmental Monitoring and Assessment, http://dx.doi.org/10.1007/s10661-011-2352-8.

2. Akgun, A., E.A. Sezer, H.A. Nefeslioglu, C. Gokceoglu and B. Pradhan. 2012. An easy-touse MATLAB program (MamLand) for the assessment of landslide susceptibility using a Mamdani fuzzy algorithm. Computer and Geoscience, 38: 23-34.

3. Althuwaynee, O.F., B. Pradhan, H.J. Park and J.H. Lee. 2014. A novel ensemble decision tree-based CHisquared Automatic Interaction Detection (CHAID) and multivariate logistic regression models in landslide susceptibility mapping. Landslides, 11: 1063-1078.

4. Ayalew, L. and H. Yamagishi. 2005. The Application of GIS -based logistic regression for landslide susceptibility mapping in the Kakuda-Yahiko Mountains, central Japan. Geomorphology, 65: 15-31.

5. Baatuuwie, N.B. and I.L. V.Leeuwen. 2011. Evaluations of three classifiers in mapping forest stand types using medium resolution imagery: a case study in the Offense Forest District, Ghana. African Journal of Environmental Science and Technology, 5: 25-36

6. Bai, S.B., J. Wang, G. Lü, P. Zhou, S.S. Hou and S.N. Xu. 2010. GIS-based logistic regression for landslide susceptibility mapping of the Zhongxian segment in the Three Gorges area, China. Geomorphology, 115: 23-31.

7. Bednarik, M., B. Magulová, M. Matys and M. Marschalko. 2010. Landslide susceptibility assessment of the Kral`ovany-Liptovsky' Mikuláš railway case study. Physics and Chemistry of the Earth, 35: 162-171.

8. Bednarik, M., I. Yilmaz and M. Marschalko. 2012. Landslide hazard and risk assessment: a case study from the Hlohovec-Sered'landslide area in south-west Slovakia. Natural Hazards, 64: 547-575.

9. Breiman, L. 2001. Random forests Machine learning, 45: 5-32.

10. Breiman, L., J. HFriedman, R.A. Olshen and C.J. Stone. 1984. Classification and regression trees. Chapman \& Hall, New York.

11. Caniani, D., S. Pascale, F. Sado and A. Sole. 2008. Neural networks and landslide susceptibility: a case study of the urban area of Potenza. Natural Hazards, 45: 55-72.

12. Catani.F., D. Lagomarsino, S. Segoni and V. Tofani. 2013. Landslide susceptibility estimation by random forests technique: sensitivity and scaling issues. Natural Hazards Earth System Science, 13: 2815-2831.

13. Cutler D.R., T.C. Edwards, K.H. Beard, A. Cutler and K.T. Hess. 2007. Random Forests for Classification in Ecology. Ecology, 88: 2783-2792.

14. Champati Ray, D.P., S. Dimri, R.C. Lakhera and S. Sati. 2007. Fuzzy-based method for landslide hazard assessment in active seismic zone of Himalaya. Landslides, 4: 101-111.

15. Chauhan, S., M. Sharma and M.A. Arora. 2010. Landslide susceptibility zonation of the Chamoli region, Garhwal Himalayas, using logistic regression model. Landslides, 7: 411-423.

16. Dai, F.C. and C.F. Lee. 2002. Landslide characteristics and slope instability modeling using GIS, Lantau Island, Hong Kong. Geomorphology, 42: 213-228.

17. Demir, G., M. Aytekin, A. Akgun, S.B. Ikizler and O. Tatar. 2013. A comparison of landslide susceptibility mapping of the eastern part of the North Anatolian Fault Zone (Turkey) by likelihood-frequency ratio and analytic hierarchy process methods. Natural Hazards, 65: 1481-1506.

18. Devkota, K.C., A.D. Regmi, H.R. Pourghasemi, K. Yoshida, B. Pradhan, I.C. Ryu, M.R. Dhital and O.F. Althuwaynee. 2013. Landslide susceptibility mapping using certainty factor, index of entropy and logistic regression models in GIS and their comparison at Mugling-Narayanghat road section in Nepal Himalaya. Natural Hazards, 65: 135-165.

19. Eemini, L., F. Catani and N. Casagli. 2005. Artificial neural networks applied to landslide susceptibility assessment. Geomorphology, 66: 327-343.

20. Gislason, P.O., J.A. Benediktsson and J.R. Sveinsson. 2006. Random forests for land cover classification. Pattern Recognition Letters, 27: 294-300.

21. Glade, T. 1997. Establishing the frequency and magnitude of landslide-triggering rainstorm events in New Zealand. Environmental Geology, 35: 160-174.

22. Hasekiogullari, G.D. and M. Ercanoglu. 2012. A new approach to use AHP in landslide susceptibility mapping: a case study at Yenice (Karabuk, NW Turkey). Natural Hazards, 63: 1157-1179.

23. Jaafari, A., A. Najafi, H.R. Pourghasemi, J. Rezaeian and A. Sattarian. 2014. GIS-based frequency ratio and index of entropy models for landslide susceptibility assessment in the Caspian forest, northern Iran. International Journal of Environmental Science and Technology, 11: 909-926.

24. Jebur, M.N., B. Pradhan, H.Z.M. Shafri, Z.M. Yusoff and M.S. Tehrany. 2015. An integrated user-friendly ArcMAP tool for bivariate statistical modeling in geoscience applications. Geoscientific Model Development, 8: 881-891.

25. Joybari, J., A. Kavian and J. Mosaffaei. 2015. Spatial and temporal variations of Tavan landslide movement in Qazvin province. Geography and environmental hazard, 4(16) (In Persian).

26. Lieb, M., B. Glaser and B. Huwe. 2012. Uncertainty in the spatial prediction of soil tex-ture: comparison of regression tree and Random Forest models. Geoderma, 170: 70-79.

27. Mohammady, M., H.R. Moradi, S. Feiznia and H.R. Pourghasemi. 2010. Comparison of the efficiency of certainty factor, information value and AHP models in landslide hazard zonation (case study: part of Haraz Watershed).Journal of Range and Watershed Management. Iranian Journal of Natural Resources, 62: 539551 (In Persian).

28. Mohammady, M., H.R. Pourghasemi and B. Pradhan. 2012. Landslide susceptibility mapping at Golestan Province, Iran: a comparison between frequency ratio, Dempster-Shafer and weights of evidence models. Journal of Asian Earth Sciences, 61: 221-236.

29. Mosaffaie, J., M. Ownegh., M. Mesdaghi and M. ShariatJafari. 2009. Comparing the efficiency of statistical and empirical landslide hazard zonation models in Alamout watershed. Journal of water and soil conservation, 16: 43-61 (In Persian).

30. Mosaffaie, J. and M. Ownegh. 2011. Landslide hazard zonation by AHP and regression model, Case study: Alamout watershed. Watershed Engineering and Management, 3: 149-158 (In Persian). 
31. Nefeslioglu, H.A., C. Gokceoglu and H. Sonmez. 2008. An assessment on the use of logistic regression and artificial neural networks with different sampling strategies for the preparation of landslide susceptibility maps. Engineering Geology, 97: 171-191.

32. Nefeslioglu, H.A., E. Sezer, C. Gökçeoğlu, A.S. Bozkır and T.Y. Duman. 2010. Assessment of landslide susceptibility by decision trees in the metropolitan area of Istanbul, Turkey. Mathematical Problems in Engineering, Article ID: 901095

33. Nicodemus, K.K. 2011. Letter to the Editor: On the stability and ranking of predictors from random forest variable importance measures. Briefings in Bioinformatics, 12: 369-373.

34. O'Brien, R.M. 2007. A caution regarding rules of thumb for variance inflation factors Quality and Quantity, 41: 673-690.

35. Oh, H.J. and B. Pradhan. 2011. Application of a neuro-fuzzy model to landslide susceptibility mapping for shallow landslides in a tropical hilly area. Computer and Geoscience, 37: 1264-1276.

36. Ozdemir, A. and T. Altural. 2013. A comparative study of frequency ratio, weights of evidence and logistic regression methods for landslide susceptibility mapping: Sultan Mountains, SW Turkey. Journal of Asian Earth Science, 64: 180-197

37. Pachauri, A.K., P.V. Gupta and R. Chander. 1998. Landslide zoning in a part of the Garhwal Himalayas. Environmental Geology, 36: 325-334.

38. Peters, J., N. Verhoest, R. Samson, P. Boeckx and B. De Baets. 2008. Wetland vegetation distribution modelling for the identification of constraining environmental variables. Landscape Ecology, 23: 10491065.

39. Pourghasemi, H.R., B. Pradhan and C. Gokceoglu. 2012a. Application of fuzzy logic and analytical hierarchy process (AHP) to landslide susceptibility mapping at Haraz watershed, Iran Natural Hazards, 63: 965-996.

40. Pourghasemi, H.R., M. Mohammady and B. Pradhan. 2012b. Landslide susceptibility mapping using index of entropy and conditional probability models in GIS: Safarood Basin, Iran. Catena, 97: 71-84.

41. Pourghasemi, H.R., M. Beheshtirad and B. Pradhan. 2014. A comparative assessment of prediction capabilities of modified analytical hierarchy process (M-AHP) and Mamdani fuzzy logic models in NetcadGIS for forest fire susceptibility mapping. Geomatics, Natural Hazards and Risk, DOI: $10.1080 / 19475705.2014 .984247$.

42. Paudel, U. and T. Oguchi. 2014. Implementation of random forest in landslide susceptibility study, a case study of the Tokamachi area, Niigata, Japan. Japan Geoscience Union Meeting, Pcaifico Yokohama, $28^{\text {th }}$ April $2^{\text {nd }}$ May, 2014.

43. Pradhan, B. 2013. A comparative study on the predictive ability of the decision tree, support vector machine and neuro-fuzzy models in landslide susceptibility mapping using GIS. Computer and Geoscience, 51: 350-365.

44. Pradhan, B. and S. Lee. 2010. Delineation of landslide hazard areas on Penang Island, Malaysia, by using frequency ratio, logistic regression, and artificial neural network models. Environmental Earth Sciences, 60: $1037-1054$.

45. Rasai, A., K. Khosravi, M. Habibnejad Roshan, A. Heidari and A. Mashayekhan. 2015. Lnadslide hazard zonation using multivariate regression in GIS environment (Case Study: Aghmashhad Watershed, Mazandaran). Watershed Management Research, 12: 205-215 (In Persian).

46. Sezer, E.A. 2010. A computer program for fractal dimension (FRACEK) with application on type of mass movement characterization Computer and Geoscience, 36: 391-396.

47. Simpson, G.L. and H.J.B. Birks. 2012. Tracking environmental change using lake sediments, Springer Publication, 5: $673 \mathrm{pp}$.

48. Stumpf, A. and N. Kerle. 2011. Object-oriented mapping of landslides using Random Forests. Remote Sensing ofEnvironment, 115: 2564-2577.

49. Toll, D.G. 1996. Artificial Intelligence Applications in Geotechnical Engineering Electronic Journal of Geotechnical Engineering, $27 \mathrm{pp}$

50.Trigila, A., F. Catani, N. Casagli, G. Crosta, C. Esposito, P. Frattini, C. Iadanza, D. Lagomarsino, S. Lari, G. ScarasciaMugnozza, S. Segoni, D. Spizzichino and V. Tofani. 2012. The landslide susceptibility map of Italy at 1:1 Million scale. Geophysical Research Abstracts, 14, EGU2012-7655, 2012.

51. Vahidnia, M.H., A.A. Alesheikh, A. Alimohammadi and F. Hosseinali. 2010. A GIS-based neurofuzzy procedure for integrating knowledge and data in landslide susceptibility mapping. Computers and Geosciences, 36: 1101-1114.

52. Vorpahl, P., H. Elsenbeer, M. Marker and B. Schroder. 2012. How can statistical models help to determine driving factors of landslides? Ecological Modelling, 239: 27-39.

53. Yalcin, A. 2008. GIS-based landslide susceptibility mapping using analytical hierarchy process and bivariate statistics in Ardesen (Turkey): comparisons of results and confirmations. Catena, 72: 1-12.

54. Yesilnacar, E.K. 2005. The application of computational intelligence to landslide susceptibility mapping in Turkey, Ph.D. Thesis Department of Geomatics the University of Melbourne, $423 \mathrm{pp}$.

55. Youssef, A.M. 2015.Landslide susceptibility delineation in the Ar-Rayth Area, Jizan, Kingdom of Saudi Arabia, by using analytical hierarchy process, frequency ratio and logistic regression models Environmental Earth Sciences, Doi: 10.1007/s12665-014-4008-9.

56. Youssef, A.M., H.R. Pourghasemi, Z.S. Pourtaghi and M.M. Al-Katheeri. 2015. Landslide susceptibility mapping using random forest, boosted regression tree, classification and regression tree, and general linear models and comparison of their performance at WadiTayyah Basin, Asir Region, Saudi Arabia Landslides, DOI 10.1007/s10346-015-0614-1.

57. Zare, M., H.R. Pourghasemi, M. Vafakhah and B. Pradhan. 2013. Landslide susceptibility mapping at Vaz watershed (Iran) using an artificial neural network model: a comparison between multi-layer perceptron (MLP) and radial basic function (RBF) algorithms. Arabian Journal of Geoscience, 6: 2873-2888.

58. Zare, M., A. Moghaddamnia, S. Tali Khoshk and H. Salmani. 2015. Landslide hazard assessment by using Neuro-fuzzy technique in Vaz Watershed. Watershed Management Research, 11: 101-110 (In Persian). 


\title{
Prioritization of Landslide-Conditioning Factors and its Landslide Susceptibility Mapping using Random Forest New Algorithm (Case Study: A Part of Golestan province)
}

\author{
Majid Mohammady ${ }^{1}$ and Hamid Reza Pourghasemi ${ }^{2}$ \\ 1- Assistant Professor, Department of Rangeland \& Watershed Management, Faculty of Natural Resources, Semnan \\ University, Semnan, Iran. Iran, (Corresponding Author: majid.mohammady@ semnan.ac.ir) \\ 2- Assistant Professor, Department of Natural Resources and Environmental Engineering, College of Agriculture, \\ Shiraz University, Shiraz, Iran \\ Received: July 16, $2016 \quad$ Accepted: October 27, 2015
}

\begin{abstract}
Landslide as a natural hazard is very dangerous especially in mountainous areas that result in loss of human life and property around the world. Iran is always exposed to landslide hazard especially in the north and west because of climatic and topographic conditions. The aim of this research is prioritization of landslide-conditioning factors and its landslide susceptibility mapping in the part of Golestan Province using random forest new algorithm. At first, landslide locations were identified using field survey, historical report, and Google earth. In total, 78 landslide locations were identified and divided into two parts for modeling (70\%) and validation $(30 \%)$. Eleven factors of landslide-conditioning including slope aspect, altitude, distance from streams, distance from faults, distance from roads, lithology, land use, slope-length, plan curvature, precipitation, and slope angle were prepared. The relationships between the effective factors and the landslide inventory map were calculated using the random forest algorithm, and then landslide susceptibility map was prepared in the GIS environment. Prioritization of landslide-conditioning factors showed that distance from road, distance from faults, and altitude have the most effect on landslide occurrence respectively. Finally landslide susceptibility map produced by random forest model were divided to four susceptibility classes such as low $(29.18 \%)$, moderate $(33.44 \%)$, high $(24.82 \%)$, and very high $(12.55 \%)$. ROC curve and the area under the curvewere used for accuracy assessment of the prepared map using about $30 \%$ of landslides. Results showed that the random forest model produced reasonable accuracy in landslide susceptibility mapping with area under curve of 0.706 .
\end{abstract}

Keywords: GIS, Landslide, Landslide susceptibility map, Random forest 